\title{
Feature Exploration for Mining Coronal Loops from Solar Images
}

\author{
Nurcan Durak, Olfa Nasraoui \\ Knowledge Discovery \& Web Mining Lab \\ Dept. of Computer Engineering and Computer Science, \\ University of Louisville \\ \{nurcan.durak, olfa.nasraoui\}@louisville.edu
}

\begin{abstract}
$^{1}$
Coronal loops are especially important in analyzing some important phenomena related to the Sun such as the controversial coronal heating problem. The analysis requires astrophysicists to manually sift through thousands of images in order to acquire images containing coronal loops. Thus, the motivation to detect these loops automatically. Since coronal loops do not have a perfect shape and are easy to confuse with other solar events, feature selection to learn characteristics of loops requires special care. In this study, we explore standard image features as well as specialized image features considering coronal loop characteristics. Our experiments confirm the success of our explored features in coronal loop detection.
\end{abstract}

\section{Introduction and Related Work}

Coronal loops are immense arches of hot gas on the surface of the Sun that have been linked to complex solar phenomena, such as violent coronal mass ejections, solar flares, and solar storms, that affect the rest of the solar system, for instance by shaking the Earth's magnetic field and power grids, and possibly harming satellites and astronomers in space. In order to make progress in studying coronal loops, scientific analysis requires data observed by instruments such as SOHO EIT ${ }^{2}$, TRACE, and SXT [1]. The biggest obstacle to complete these studies has been manually

\footnotetext{
1 This work is supported by the National Aeronautics and Space Administration under Grant No. AISR-03-0077-0139 issued through the Office of Space Sciences and by National Science Foundation Grant IIS-0431128.

2 EIT: Extreme ultraviolet Imaging Telescope on board Solar and Heliospheric Observatory (SOHO): http://umbra.nascom.nasa.gov/eit/
}

putting the relevant data sets together prior to any scientific analysis. Instead of manually sifting all the images, an automated system, that retrieves only images with coronal loops, would make scientific progress easier and faster. To retrieve those images from SOHO's EIT image collection, the characteristics of coronal loops should be analyzed. In this study, we especially concentrate on the feature extraction during the coronal loop learning phase of a system for automated retrieval of images with coronal loops.

Coronal loop shapes have several unique characteristics, with great diversity in their sizes, intensity distributions along the arch, and orientations. For example, Figure 1(a) shows a big and strong loop, while Figure 1 (b) shows a small and faint loop that may disappear after applying preprocessing techniques. Figure 1(c) shows a coronal loop in a noisy region, and under a similar solar event known as prominence. Distinguishing a single loop shape amidst a cluttered region can be hard due to similar intensity values all around the loop shape. In addition to their diversity, the majority of loop outlines are incomplete, with portions of the loop typically fading away, or intensity levels along the loop decreasing after a certain point along the loop. This causes loss of image quality in the loop arch.
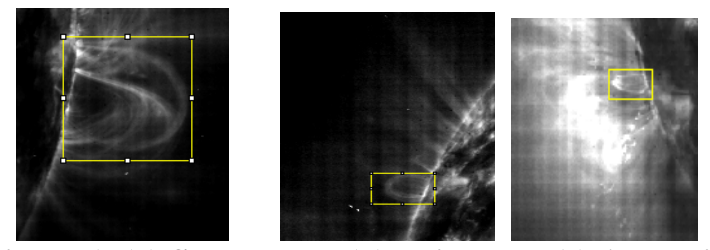

Figure 1. (a) Strong loop (b) Faint loop (c) A loop in noisy region

Besides the problems that are directly related to the loop shapes, we faced another big challenge when we consider that the corona can have other kinds of events 
(e.g. coronal mass ejections and solar flares). The similarity between the characteristics of coronal loops and the other events tends to confuse automated classifiers. In fact, distinguishing them could be difficult even for the Human eye. Figure 2 shows two examples of such other events similar to coronal loops.

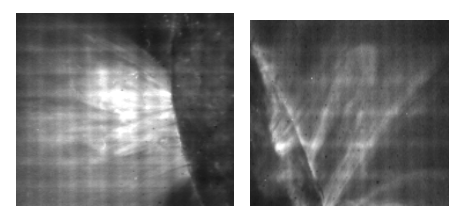

Figure 2. No-loop regions

Selecting and discovering appropriate features to capture the characteristics of loop shapes and non-loop shapes play an important role in automatic coronal loop detection. In this study, we investigate several standard image features such as Hough lines [2], statistical features [3], edge histogram descriptors [4], as well as specialized features that we have developed to handle the complicated nature of coronal loops such as specialized curvature features or spatial features.

There is little work investigating image features to detect coronal loops in the current literature. In [5], loops were traced in a region that was already known to contain a loop, rather than detected automatically without even knowing their presence as in our case. In [6], features for other kinds of solar events such as sun spots, filaments, plages, or solar flares have been surveyed. However neither of those events shares any similarities with coronal loops, making the features explored for other events ineligible for our problem.

The rest of the paper is organized as follows. Section 2 presents the steps in the image preparation, before the feature extraction, which is explained in Section 3. Section 4 presents our experimental evaluations, and Section 5 concludes this paper.

\section{Image Preparation}

To classify solar images according to the occurrence of loop shapes, FITS images are first downloaded from SOHO's EIT image repository in the $171 \mathrm{~A}^{\mathrm{o}}$ wavelength (best for viewing loops). EIT images are $1024 \times 1024$ in size and gray level. A sample EIT image, with two expert-marked coronal loops, is shown in Figure 3. To improve the quality of downloaded images, image preprocessing techniques, such as noise removal or contrast enhancement techniques [3], are first applied. After preprocessing, we divide the solar circumference into regions that we call 'blocks'. A block is defined by its position, height and width values. After generating several blocks from each image, these blocks are labeled as either containing solar loops (i.e., Loop class), or not (i.e. NO-loop class). The average number of blocks in an image ranges between 50 and 60, and if there is a marked loop in an image, it typically spreads over two to four consecutive blocks. The average ratio of Loop blocks to NO-Loop blocks is around 1 to 20, which means that we are also dealing with an imbalanced class data set. After extracting blocks, we are ready to extract features from blocks to distinguish loop regions from no-loop regions.

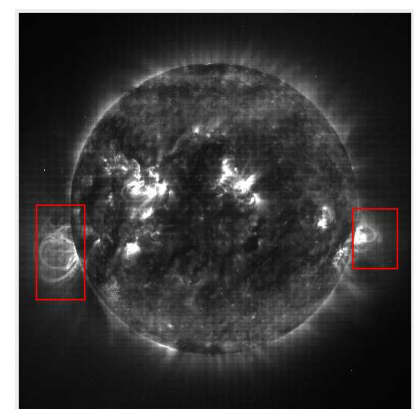

Figure 3. Sample EIT image

\section{Feature Extraction}

The features are extracted from each block separately. Since features extracted from only the intensity level blocks are not sufficient to recognize loop shapes, we extract additional features from binary blocks that are obtained after applying edge detection and global thresholding [3] on the blocks. Figure 4 shows a sample of both the pre-processed intensity version and the binary version of the same block.
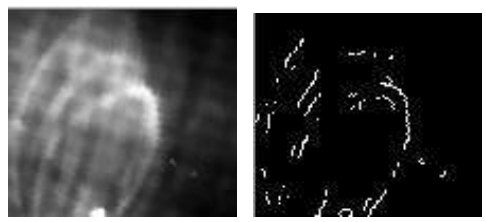

Figure 4. (a) Intensity level block. (b) Binary block

From the intensity level blocks, the following statistical features [3] are extracted: Mean, Standard Deviation, Smoothness, Third Moment, Uniformity, and Entropy. The remaining features are extracted from the binary level blocks. The "Number of Edge Pixels" is the total number of pixels located on all the edges in the binary blocks. Since the distribution of line segments seemed to be an indicative feature, we count the number of lines in the blocks, after using the Hough Transform [2]. The line lengths can be estimated by 
counting the number of intersecting sinusoidal curves in the peak corresponding to the line. Thus from the Hough Space, we acquire two features: "Number of Hough Lines" and "Length of Longest Hough Line" which is the number of points in the global maximum spot of the Hough Space.

The directions of lines in the blocks also seemed to be good discriminating features. The standard Edge Histogram Definition (EHD) descriptors [4] were not satisfying in our case, because the extracted line orientations were hard to match exactly to the standard $0^{\circ}, 45^{\circ}, 90^{\circ}, 135^{\circ}$ angles. Thus, we obtained the following modified edge histogram features by mapping the edge angles $\theta$ into more tolerant ranges: Number of Vertical Edges: $\theta$ in $80^{\circ}-100^{\circ}$, Number of Horizontal Edges: $\theta$ in $0^{\circ}-10^{\circ}$ or in $170^{\circ}-180^{\circ}$, Number of $45^{\circ}$ Edges: $\theta$ in $35^{\circ}-55^{\circ}$, Number of $135^{\circ}$ Edges: $\theta$ in $125^{\circ}-145^{\circ}$, Number of Non-Directional Edges: $\theta$ not in any of the above ranges.

Because we observed that the edges tend to be mostly confined to the bottom of the No-loop blocks (e.g. Figure 5 (a)), whereas in loop blocks, the edges were mostly spread out all the way to the top part of the loop block (e.g. Figure 5 (b)), we decided to calculate the spatial information of the edge distribution in the blocks by dividing the block into four horizontal bands, and counting the number of edge points in each band. Thus, each band's edge distribution gives us spatial features, called First Band Edges, Second Band Edges, Third Band Edges and Fourth Band Edges.

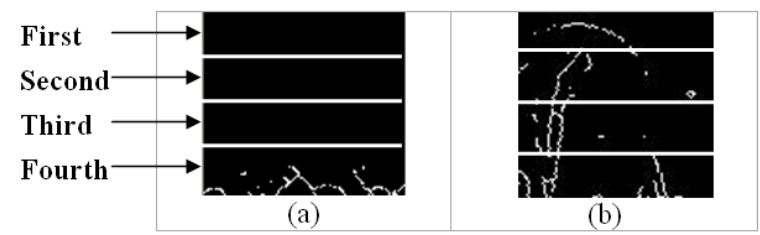

Figure 5: Four bands of the blocks (a) No-Loop Block (b) Loop Block

Taking into account all the problems associated with loop shapes, we developed our own curvature feature generation algorithm. First we automatically trace the curves considering their noisy and defective nature. Tracing a curve starts from an initial point that is propagated by adding new points. The next point to be added to the curve structure is selected from nearby points $[6,7]$. In this phase, a wrong selection may lead to undesired paths. Therefore defining a cost function for each candidate point should be done very carefully. We pay attention to both orientation similarity and distance factors for choosing the best continuation point. After tracing curve segments, we investigate their relationship to each other. If their endpoints are in the same vicinity and these endpoints share the same angle range $[-\theta,+\theta]$ for the orientation, we assume that these segments are part of a single curve structure. Figure 6 shows an example output of our curve tracing approach.

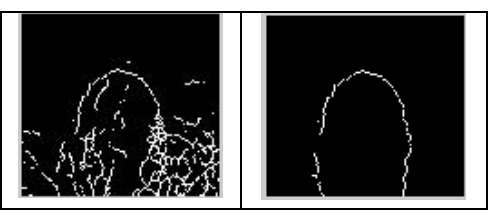

Figure 6. (a) Original image (b) Automatically traced curve

Since we have no prior information about the presence of a loop, we apply our curve tracing algorithm on all regions, both with and without loops. Since the non-loop regions also have some curvilinear shapes, our curve tracing algorithm may end up with some line groups from these regions. To distinguish these lines from the desired curves, we introduce the 'Curvature Strength Feature' which measures how curvy an extracted curve is. The strength of a curve depends on the radius, length, and angle difference along the curve. This feature can help distinguish regions with curves (e.g. Figure 7 (a)) from regions without any curve (e.g. Figure 7 (b)).
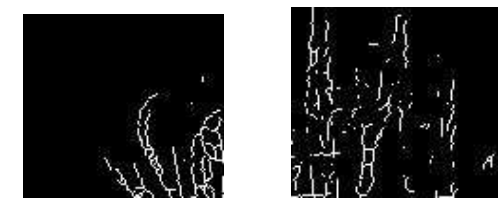

Figure 7. (a) A region with a curve, (b) A region without a curve

In addition to the 'Curvature Strength', we extract other useful features from the traced curves. We use the length of the traced curve, called 'Curve Length', and also use the 'Number of Segments' which are detected during the curve segmentation process. Dominant points are the points located between two curve segments whose directions are different from each other [7]. We also use the 'Sign Distribution' along the traced curve as a feature. If there are both positive signs which are continuation points going upward and negative signs which are continuation points going downward along the edge, the possibility of existence of a curve structure is higher than if the edges consisted of only one kind of sign. Figure 8 illustrates positive and negative signs along a curve. Another useful feature about curves was the 'Peak Angle' which is computed using two different line equations corresponding to the two traced sub-segments/sides of 
the traced curve ( $\alpha$ in Figure 8). If these lines intersect, then the angle between the two lines is considered as a 'Peak Angle'. The last feature related to curvature is the 'Projected Curve Length' ( $d$ in Figure 8) which is the Euclidean distance between the two endpoints of the entire curve structure.

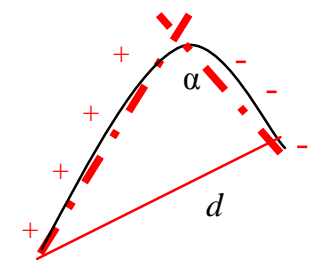

Figure 8. 'Sign Changes', 'Peak Angle' $\alpha$, and 'Projected Curve Length', $d$ on an artificial curve

\section{Experimental Results}

By considering various types of loop shapes and no-loop shapes, we constructed our current training data set which contains 403 Loop blocks and $7200 \mathrm{No}$ loop blocks. After extracting all the features discussed so far, we applied the rank search algorithm on all the explored features according to the information gain measure [8]. As shown in Figure 8, the features, sorted in descending order of their information gain are: Curve Length, Number of Edge Pixels, Peak Angle, Curvature Strength, Length of Longest Hough Line, Sign Distribution, Projected Curve Length, Third Band Edges, etc. The information gain measure confirms that the specialized loop features are better than standard statistical features.

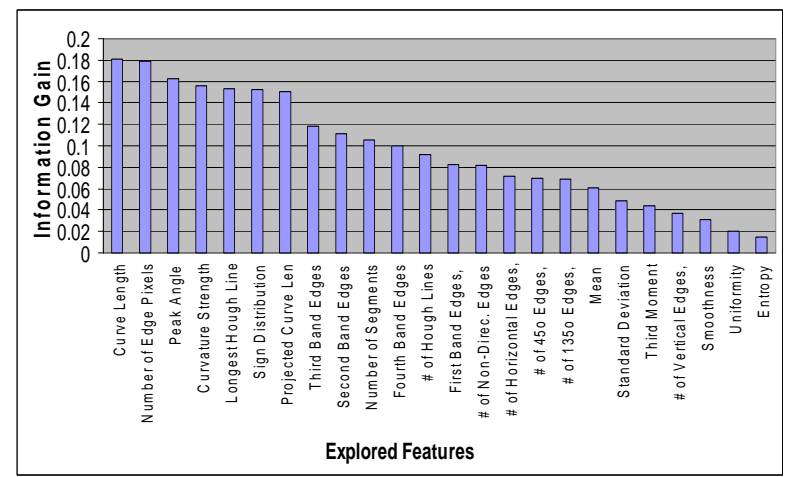

Figure 8. Information gain for the investigated features

We trained our system using all the investigated features with several known classifiers [8] including Adaboost (with $\mathrm{C} 4.5$ as a base classifier), Naïve Bayes (NB), Multi-Layer Perceptron (MLP), C4.5 decision trees, Ripper, and k-Nearest Neighbors (k-NN). We performed 10 -fold cross-validation to evaluate the performance on this data set. Table 1 shows the precision and recall value pairs for different classifiers. The best results are $62 \%$ precision and $69 \%$ recall values, which are significantly better than random guessing because of the severe imbalance of the data (roughly 0.5 to 10 for the loop class).

Table 1: Classifier results

\begin{tabular}{|l|l|l|}
\hline Classsifier & Precision & Recall \\
\hline AdaBoost(C4.5) & 0.63 & 0.662 \\
\hline NB & 0.363 & 0.768 \\
\hline MLP & $\mathbf{0 . 6 2 1}$ & $\mathbf{0 . 6 9 4}$ \\
\hline C4.5 & 0.568 & 0.563 \\
\hline RIPPER & $\mathbf{0 . 6 2 3}$ & $\mathbf{0 . 6 9 6}$ \\
\hline K-NN (k=5) & 0.644 & 0.615 \\
\hline
\end{tabular}

\section{Conclusion}

For the automated classification of coronal loop regions, we investigated a wide range of features. Of the 24 features, 6 were standard intensity-based features, and 18 were specialized features, that considered the coronal loop characteristics. We obtained the best information gain values from the specialized features. The classification results using the investigated features and a variety of classifiers gave promising results in the automatic detection of coronal loops from SOHO's EIT image data.

\section{References}

[1] J.T. Schmelz, R.T. Scopes, and J.W. Cirtain. Determining Coronal Heating of Plasma Loops Through Differential Emission Measure Analysis. Adv. in Space Research, 30 (3): 507-516, 2002.

[2] R. O. Duda, and P. E. Hart. Use of the Hough Transformation to Detect Lines and Curves in Pictures, Comm. A3CM, Vol. 15: 11-15 (1972).

[3] R.C. Gonzalez, R. Woods. Digital Image Processing. Prentice Hall, 2/E, 2002.

[4] C.S Won, D.K. Park, S.J. Park. Efficient Use of MPEG-7 Edge Histogram Descriptor. ETRI Journal, 24 (1): 23-30, 2002.

[5] J.K Lee, T.S. Newman, G.A. Gary. Oriented connectivity-based method for segmenting solar loops. Pattern Recognition, 2006.

[6] V. Zharkova, S. Ipson, A. Benkhalil, and S. Zharkov, Feature recognition in solar images, Art. Int. Rev., 23/3, 209266, (2005)

[6] K. Raghupathy, T.W. Parks. Improved Curve Tracing in Images. ICASSP 2004.

[7] W.Y. Wu, An adaptive method for detecting dominant points, Pattern Recognition, Volume 36, Number 10: 2231-2237, 2003

[8] T.M. Mitchell, Machine Learning. The Mc-Graw-Hill Companies, Inc., (1997) 